Referencia para citar este artículo: Nava-Preciado, J. M., Méndez-Huerta, M. \& (2019). Adolescentes y derechos humanos: una aproximación a su ejercicio efectivo en la escuela. Revista Latinoamericana de Ciencias Sociales, Niñez y Juventud, 17(1), 285-299. doi:https://dx.doi.org/10.11600/1692715x.17117

\title{
Adolescentes y derechos humanos: una aproximación a su ejercicio efectivo en la escuela*
}

\author{
José María NAVA-PRECIADO ** \\ Profesor Universidad de Guadalajara, México. \\ MAURICIO MÉNDEZ-HUERTA *** \\ Profesor Universidad de Guadalajara, México.
}

Artículo recibido en febrero 13 de 2018; artículo aceptado en abril 20 de 2018 (Eds.)

- Resumen (analítico): este escrito es parte de una investigación cuyo objetivo es analizar el conocimiento de alumnas, alumnos, profesoras y profesores sobre la aplicación efectiva de la Ley General de los Derechos de Niñas, Niños y Adolescentes en el espacio escolar, a tres años de su publicación en México. Es un estudio cualitativo, realizado en escuelas secundarias públicas de la ciudad de Guadalajara. La información se recuperó mediante seis grupos de discusión con adolescentes y un cuestionario aplicado a 84 docentes. Los resultados indican que el desconocimiento de la ley se vincula de manera importante con el ejercicio no eficaz de los derechos humanos. A mayor desinformación de los agentes, mayor inclinación del profesorado a vulnerar los derechos del alumnado en el aula y menor capacidad de este para exigirlos.

Palabras clave: derechos humanos, escuela secundaria, aplicación de la ley, adolescencia, docente de secundaria (Tesauro de Ciencias Sociales de la Unesco).

\section{Adolescents and human rights: an approximation of the effective exercising of rights in school}

- Abstract (analytical): This paper is part of a research study aimed at analyzing the knowledge of male and female students and teachers on the effective application of the General Law for the Rights of Children and Adolescents in the school, three years after this law came into effect in Mexico. This was a qualitative study implemented in public junior high schools in the city of Guadalajara. The information was obtained through holding six discussion groups with adolescent students and conducting a questionnaire survey with 84 teachers. The results indicate that ignorance of the law is highly linked with the non-effective exercising of children and adolescents' human rights. The study shows that as the amount of misleading information provided to students increases, it is more

Este artículo de investigación científica y tecnológica es parte de los resultados de una investigación científica que se inicia en febrero de 2017 y finaliza en mayo de 2018, titulada «Derechos humanos: el ejercicio efectivo, su aplicación y análisis en adolescentes», desarrollada dentro del Cuerpo Académico Adolescentes: mundo y vida, clave UDG-CA-967, auspiciada por el Departamento de Filosofía de la Universidad de Guadalajara, en el marco de la convocatoria 7.1-2017. Área de conocimiento: Otras Humanidades, subárea: filosofía.

** Doctor en Educación por la Universidad Pedagógica Nacional (UPN). Profesor titular «C» del Departamento de Filosofía de la Universidad de Guadalajara, miembro del SNI, nivel I. Researcher ID: L-5260-2017. Orcid: 0000-0003-2050-1251. Índice H5: 1. Correo electrónico: jnava_ preciado@yahoo.com.mx

*** Maestro en Lingüística Aplicada por la Universidad de Guadalajara. Profesor asociado «A» del Departamento de Filosofía de la Universidad de Guadalajara, miembro del Cuerpo Académico Retórica. Lógica y Teoría de la Argumentación. ReasercherID: N-9554-2017. Orcid: 0000-00027283-8051. Correo electrónico: mendezhuertamauricio@hotmail.com 
probable that teaching staff violate the rights of this population in the classroom, and male and female students have less capacity to demand the fulfilling of their rights.

Key words: Human rights, junior high school, law enforcement, adolescence, junior high school teacher (Unesco Social Sciences Thesaurus).

\section{Adolescentes e direitos humanos: uma aproximação ao seu exercício efetivo nas escolas}

Resumo (analítico): este artigo faz parte de uma pesquisa que tem por objetivo analisar o conhecimento de alunas, alunos, professoras e professores sobre a aplicação efetiva da Lei Geral dos Direitos de Meninas, Meninos e Adolescentes no espaço escolar, três anos após sua publicação no México. Trata-se de um estudo qualitativo, realizado em escolas secundárias públicas da cidade de Guadalajara. A informação foi obtida através de seis grupos de discussão com adolescentes e um questionário aplicado a 84 professores. Os resultados indicam que o desconhecimento da lei está ligado de maneira importante ao exercício ineficaz dos direitos humanos. Quanto maior a desinformação dos agentes, maior a inclinação dos professores a violar seus direitos na aula e menor a capacidade dos adolescentes para exigi-los.

Palavras-chave: direitos humanos, escola secundária, aplicação da lei, adolescência, professor de secundária (Thesaurus de Ciências Sociais da Unesco).

\section{-1. Introducción. -2. Plataforma metodológica. -3. Resultados. -4. Discusión. -5. Conclusión. -Lista de referencias.}

\section{Introducción}

La defensa de los Derechos Humanos (DH) de niñas, niños y adolescentes no está exenta de dificultades y polémicas contradicciones (Lozano-Vicente, 2016). En los debates que lleva a cabo la sociedad mexicana, resulta incuestionable la premisa de reconocer los derechos de este segmento de la población. Así, se presume que este grupo social debe crecer en ambientes de amor, bienestar y comprensión para lograr su pleno desarrollo, pero el reconocimiento de tales derechos no garantiza su ejercicio en términos prácticos. Determinar el cumplimiento efectivo de los $\mathrm{DH}$ de niñas, niños y adolescentes implica mantener a este grupo y a los responsables de su observancia en un constante proceso deliberativo, justificado como necesario ante los desajustes sociales y estilos de vida que confrontan un mundo moderno, contaminado por la violencia (Bauman \& Donskis, 2015).

En contextos culturales y sociales diferenciados, los menores siguen siendo víctimas de varios tipos de violencia (Comisión Nacional de los Derechos Humanos - CNDH—, 2015), pobreza (Silva \& Martínez-Guzmán, 2017) y exclusión (Hernández, Cardona-Arango, Segura-Cardona, 2018). Por ejemplo, en el estudio hecho en México por Fuentes, González, Padrón y Tapia (2015) se puede ver que los DH de las niñas, niños y adolescentes se limitan por las formas de entender y aproximarse a este grupo de población. Así mismo, en su informe especial 2017 (publicado en 2018), la CNDH presenta las condiciones de vulnerabilidad en que vive una porción significativa de adolescentes en el país, relacionadas con asuntos de pobreza, salud, educación y empleo, permeados por cuestiones étnicas y de género (Comisión Nacional de los Derechos Humanos, \& Centro de Investigaciones y Estudios Superiores en Antropología Social, 2017). En estos estudios se observa que el ejercicio efectivo de sus derechos no está resuelto y que México no cumple a cabalidad con los mandatos para salvaguardar sus DH. Resulta obvio que, al vivir estas experiencias, las personas adolescentes ${ }^{1}$ se convierten en víctimas; débiles y vulnerables, difícilmente enfrentarán con éxito los desafíos de un

1 En México, la LGDNNA engloba los DH de (1) niñas y niños y (2) adolescentes. Pero, siguiendo a Matus (2013), cada etapa de la vida tiene sus propias particularidades, porque sus necesidades son heterogéneas. Consecuentemente, conocer estos dos segmentos de población, supone investigaciones diferenciadas. Así, aclaramos que, por cuestiones de interés, nuestro estudio solo se centra en los adolescentes, pese a que la LGDNNA regula los derechos de ambos grupos. 
mundo que los reclama activos y responsables en la demanda de sus prerrogativas. Su proyecto de vida se trastoca cuando sus derechos resultan afectados; de ahí la importancia social de obstaculizar lo menos posible su crecimiento personal, cultivando su sensibilidad moral y cívica. Estos agentes morales deben concebirse como verdaderos protagonistas de sus derechos y como sujetos activos de su propia realidad (Ravetllat-Ballesté, 2016).

En México, la Ley General de los Derechos de Niñas, Niños y Adolescentes (LGDNNA) entra en vigor el 4 de diciembre de 2014, dentro del marco de las reformas constitucionales del país (CNDH, 2015). Vale la pena señalar que las anteriores leyes en materia de protección de los DH de este segmento de población tenían un carácter más bien privativista, y con la LGDNNA se busca que el Estado actúe como un agente activo y promotor de los DH en el marco del modelo convencional garantista (González, 2016). La LGDNNA impone deberes y obligaciones para la sociedad, lo que implica su puesta en práctica por todos los miembros de aquella. Nussbaum (2006) sostiene que cualquier sociedad decente debe responder a las necesidades de los grupos vulnerables con educación y respeto; esto es así porque, a pesar de que la gran mayoría de las niñas, niños y adolescentes son personas con capacidades plenas, no logran llenar muchos requisitos para su desarrollo.

Es en la familia y la escuela, esos espacios cotidianos y domésticos, donde más se presenta una privación sistemática de los $\mathrm{DH}$ de las personas adolescentes, como reflejo de prácticas educativas tradicionales, en las que padres y docentes presumen que para educarlos se debe recurrir a dispositivos como el poder y el castigo. Así, a nuestro juicio, se deben dar pasos firmes en la aplicación irrestricta de la ley. Conforme a este principio, la escuela y la familia no pueden ser excepciones al ejercicio eficaz de los DH que se plantea en la citada ley, pues fallaríamos al pensar que hay agentes exonerados de su observancia: docentes y familiares. Esto implica suponer que la violación de los DH tiene, en muchos casos, un rostro familiar.

En la ley subyace el imperativo de que siempre - y en cualquier circunstancia - debe ponderarse el interés de los agentes morales, salvaguardando su integridad. En ese sentido, es pertinente aclarar que, cuando pensamos en las personas adolescentes, la deliberación se relaciona con el desarrollo integral de su personalidad y permite reconocer que existen restricciones para garantizar su pleno crecimiento. Sin embargo, todo carácter restrictivo debe ser razonable y proporcionado, como afirma Bernal (2008); por ejemplo, siempre debemos justificarlo en función de lo que es más conveniente jurídica y éticamente, hablando para ellas y ellos.

Es evidente que la dimensión jurídica es fundamental para el reconocimiento de los $\mathrm{DH}$, pero lo importante en estos momentos es la exigencia de su materialización; particularmente, los espacios locales constituyen lugares privilegiados para hacer efectiva la garantía de los derechos de niñas, niños y adolescentes, como lo sostiene en su estudio Durán-Strauch (2017). Por su parte, Calvinho (2012) arguye que el reconocimiento y la declaración de los DH son letra muerta si no se posibilitan dispositivos para su observancia. De igual manera, Anaya (2008) y García-Cívico (2010) consideran que una de las dimensiones fundamentales de los DH es definir indicadores que permitan medir si es efectivo o no su ejercicio, considerando las diferentes y diferenciadas variables políticas, culturales y sociales prevalecientes en el entorno. Así mismo, la preocupación por su observancia debe darse en el tránsito real de su existencia (Silva, 2017); a veces ni la sociedad ni las personas adolescentes son conscientes de ello, por lo que se permiten conductas y prácticas que menoscaban sus derechos, con el argumento de que hay que educarlas. Por tanto, las deliberaciones sobre los DH de este grupo de población deben hacerse a partir de sus propias experiencias y en la praxis diaria (que se vuelve costumbre y se distancia de los meros formalismos de la ley).

Así, tenemos una norma que protege y promueve los derechos de este grupo social, pero al mismo tiempo existe la privación de sus derechos en diversos ámbitos, razón por la que justificamos algunos interrogantes: ¿las personas adolescentes conocen esos derechos? ¿Cómo los viven? ¿Se ven como sujetos activos de sus derechos? ¿El profesorado tiene conocimiento de tales derechos? Estas preguntas nos invitan a poner en práctica la buena deliberación (Aristóteles, 2012), misma que, grosso modo, consiste en someter los juicios de las personas participantes a procesos de interpelación 
con sus interlocutores, según el contexto; dicho desplazamiento de carácter retórico nos ayuda, por su naturaleza práctica, a comprender la centralidad del otro al momento de dialogar.

De esta manera, el objetivo del presente escrito es poner de relieve los juicios y posiciones de un grupo de adolescentes, profesoras y profesores acerca de sus experiencias con la LGDNNA, para explorar el ejercicio efectivo de dicha ley en el espacio escolar.

\section{Método}

Naturaleza del estudio. A partir de los principales enfoques epistemológicos y metodológicos en la investigación, nuestro estudio está orientado al paradigma cualitativo. Este modelo propone cierto grado de flexibilidad en relación con el objeto de investigación (Ragin, 2007) por dos razones interrelacionadas: 1) permite dar voz a las personas dentro de un contexto deliberativo; 2) la realidad se define a través de las interpretaciones de los participantes de la investigación respecto a sus propias mediaciones (Hernández, Fernández, \& Baptista, 2014). En lo referente a sus fines, nuestra investigación es de carácter exploratorio; según Yuni y Urbano (2014), esta perspectiva se caracteriza por tratar un tema del cual no se tiene demasiado conocimiento, por lo que la información obtenida de la realidad social es de carácter preliminar y exploratorio.

Sujetos. Flick (2007) plantea que en la investigación cualitativa las decisiones de muestreo se dirigen a personas o grupos que prometen mayores ideas e información relevante. Desde este criterio epistemológico, nuestro análisis se centra en dos grupos ${ }^{2}$ receptores de la LGDNNA, que comparten la escuela secundaria como espacio de convivencia: 1) las personas adolescentes, sujetos titulares de derechos según la ley; 2) el profesorado considerado como corresponsable en promover y proteger los DH. ¿Por qué elegir adolescentes estudiantes de secundaria? Porque, según la Secretaría de Educación Pública (SEP, 2017), la educación secundaria es crucial como espacio formativo en la vida de las personas jóvenes, motivo por el cual lo que sucede dentro de ella debe estar bajo la mirada de la sociedad (Garzón, 2005), y porque en esta etapa las personas forman su sensibilidad y juicio moral, elementos básicos para una adecuada integración a sus comunidades.

Criterios para la elección de las escuelas. Desde el criterio de un muestreo intencional se escogieron escuelas de la ciudad de Guadalajara, puesto que una de sus características es elegir, entre otras cosas, una muestra de sujetos a los que se tiene fácil acceso (Sáez, 2017). Igualmente, optamos por secundarias públicas porque en nuestro contexto presentan características relevantes para los propósitos del estudio: a) asisten estudiantes de escasos recursos; b) los grupos son numerosos, por lo general; c) carecen de un buen mantenimiento; d) el contexto que las rodea es adverso.

Así, dados estos criterios, se trabajó con seis escuelas de una zona escolar de Guadalajara, enclavadas en barrios populares de la ciudad. En ellas, según el inspector de la zona escolar, el $60 \%$ de sus estudiantes provienen de lo que él denomina «familias disfuncionales». En el caso de las estudiantes y los estudiantes, intencionalmente se eligió un grupo de tercer año por plantel, ya que al llevar tres años en la secundaria tienen la experiencia para evaluar la cultura de los DH en su escuela, mientras que, por criterios de accesibilidad, se trabajaría con las docentes y los docentes presentes al momento de visitar las instituciones.

Técnicas para recuperar la información. A partir de la necesidad de obtener empíricamente información válida y confiable (Yuni \& Urbano, 2014), definimos los grupos de discusión y la encuesta como las herramientas más apropiadas para conseguirla; la primera con estudiantes y la segunda con la comunidad de profesores, considerando los objetivos del trabajo. El grupo de discusión es una herramienta que pone en juego el arte de la buena deliberación, porque las personas debaten sobre problemas cercanos en un contexto que posibilita analizarlos y evaluarlos frente a la mirada crítica de los otros para lograr acuerdos. Las creencias, muchas veces prejuiciosas, se someten al escrutinio del grupo hasta llegar a un consenso o disenso razonado. Deliberamos sobre las cosas que están

2 Conviene señalar que los destinatarios directos de la ley son niñas, niños y adolescentes. De acuerdo con los propósitos del estudio, concebimos a las docentes y los docentes como destinatarios indirectos porque, en más de algún sentido, su tarea es promover que se cumpla el mandato de la ley. 
cercanas y sobre aquello que puede elegirse y hacerse, «que son, de hecho, las que restan por decir» (Aristóteles, 2012, p. 54). Así, las individualidades y particularidades que lo componen (Suárez, 2005) se convierten en juicio fundamentado y calculado.

Por su parte, la encuesta ${ }^{3}$ se utilizó como instrumento de registro para obtener información del profesorado sobre la LGDNNA, con el fin de triangular posteriormente su postura con las valoraciones arrojadas por las discusiones con el alumnado. El análisis pormenorizado de la ley sirvió como marco analítico para construir los ítems del instrumento. El protocolo seguido para el trabajo de campo fue el siguiente: a) se presentó el proyecto de investigación a directivos de las seis escuelas que integran la zona escolar, en una reunión de trabajo solicitada por el inspector; b) para la conformación de los grupos de discusión, se pidió a la dirección de cada escuela que los estudiantes y las estudiantes fueran de tercero y que el grupo de trabajo estuviera integrado por cuatro mujeres y cuatro hombres de salones diferentes. Así, se organizaron seis grupos deliberativos - tres en el turno matutino y tres en el turno vespertino - en el mes de junio de 2017, justo al finalizar el año escolar, porque partimos del supuesto de que, después de tres años, las personas jóvenes conocen ampliamente las dinámicas institucionales y pueden emitir un juicio valorativo sobre su estancia escolar, antes de abandonar la escuela y continuar con su siguiente etapa formativa; c) para aplicar el instrumento a la comunidad docente, se contó con una persona comisionada por la dirección de la escuela y solo se administró a las profesoras y profesores presentes en ese momento, recuperándose apenas 84 cuestionarios.

Tratamiento de la información. Debido al enfoque del estudio, se cuidó específicamente que el dato emergiera en los grupos de discusión y a partir de su análisis se codificaron las valoraciones de los sujetos de investigación, siguiendo la estrategia recomendada por Flick (2007), triangulando las similitudes de sus posiciones y construyendo unidades semánticas que originaron una serie de categorías (tabla 1). Estas se depuraron y aclararon conforme se analizaba el material empírico, siempre tomando como referente la LGDNNA. «La investigación cualitativa implica a menudo un proceso de aclaración recíproca entre la imagen que tiene el investigador del objeto de investigación, por un lado, y los conceptos que enmarcan la investigación, por el otro» (Ragin, 2007, p. 145). De los datos obtenidos del instrumento aplicado a profesoras y profesores, agrupamos la frecuencia y distribución de sus respuestas para posteriormente triangular la información con las unidades de registro de la gente entrevistada y confrontarlas con las categorías señaladas. En este trabajo se integran los resultados por considerarlo apropiado y se muestran fragmentos relevantes, pero coincidentes, que ilustran la postura de las jóvenes y los jóvenes de las escuelas en cada eje de discusión ${ }^{4}$.

Tabla 1. Construcción de categorías a partir del material empírico

\begin{tabular}{|l|l|}
\hline \multicolumn{2}{|c|}{ Categorías } \\
\hline $\begin{array}{l}\text { (1) La escuela como promotora de los } \\
\text { derechos en general. }\end{array}$ & $\begin{array}{l}\text { (5) Del derecho a tener un ambiente } \\
\text { favorable. }\end{array}$ \\
(2) Del derecho a tener información & (6) Del derecho a no ser discriminado. \\
para el desarrollo de su personalidad. & (7) Del derecho a que se respete la \\
(3) Del derecho a contar con espacios & diversidad. \\
dignos. & (8) Del derecho a que su opinión se \\
(4) De las medidas disciplinares de la & tome en cuenta en asuntos escolares. \\
escuela. & \\
\hline
\end{tabular}

3 Es importante señalar que construimos nuestro instrumento tomando como ejemplo la Encuesta Nacional de Niños, Adolescentes y Jóvenes que investigadores de la Unam utilizaron en el estudio «Los mexicanos vistos por sí mismos. Los grandes temas nacionales» (Córdova, Flores, Alejandre, \& Vázquez del Mercado, 2015). Una vez diseñado, se puso a consideración de colegas expertos en estudios de énfasis cuantitativos para su validación. Hechas las adecuaciones sugeridas, se usó como instrumento en un pilotaje de quince profesoras y profesores de una secundaria de contexto similar a los planteles en los que aplicaríamos la encuesta.

4 Aquí optamos por códigos que constan del grupo de discusión correspondiente y del número de la unidad de información. 


\section{Resultados}

Dentro del currículo formal, la escuela secundaria tiene la materia de Formación Cívica y Ética, conocida como Force, que se ha convertido — según los grupos de estudiantes — en el espacio propicio para conocer sus derechos:

E: ¿En la escuela les dicen que tienen derechos humanos?

Todos: ¡Sí!

$\mathrm{E}$ : ¿Cómo les informan?

Aa: En la materia de Formación Cívica y Ética.

$\mathrm{E}:$ ¿Y los otros maestros?

Aa: Los otros maestros no.

(Grupo A.003)

$\mathrm{E}:$ ¿Ustedes saben que tienen derechos humanos?

Aos: ¡Sí!

E: ¿Cómo lo saben?

Ao: Por la materia de Formación Cívica y Ética.

Aa: Nos lo han inculcado desde la primaria.

$\mathrm{E}:$ ¿Cómo?

Ao: Mediante la materia de Cultura de la Legalidad.

(Grupo C.002)

$\mathrm{E}:$ ¿Les dan pláticas sobre derechos humanos?

Aa: Mmm... Tenemos... Es el tema por la clase de Force, pero de ahí en más no tenemos pláticas de derechos humanos; para nosotros no.

E: ¿Y los demás? ¿Son del mismo grupo?

Aos: No.

Ao: Como una clase específica o algo así no tenemos, no más lo que viene proporcionado por el libro y los temas que vemos en la materia de Force. Pero de otra cosa aparte no.

(Grupo D.011)

Como se observa, los DH son tratados en la escuela de manera marginal, pese a ser un tema indispensable dado nuestro contexto social marcado por la violencia. Este y otros temas emergentes son poco atendidos dentro del currículo escolar de educación básica, según el Instituto Nacional para la Evaluación de la Educación (2016). Así, respecto a si conocen la LGDNNA, los seis grupos de trabajo dicen no conocerla. No obstante, las y los estudiantes enumeraron algunos de sus derechos. Entre los más mencionados están: 1) educación, 2) tener una familia, 3) salud y sexualidad, 4) diversión, 5) libertad de expresión, 6) igualdad. Estos están contemplados en la lista de los 20 derechos enumerados por la LGDNNA.

Ante este panorama, se hace imperativo explorar si la educación secundaria le garantiza al alumnado el derecho a la información sobre aspectos relacionados con sus intereses. ¿Cumple la escuela con esa tarea? ¿Brinda información idónea y suficiente sobre temas como la sexualidad, el uso responsable de las tecnologías de la información, las adicciones y la orientación vocacional? En la narrativa de la comunidad escolar entrevistada se observa el siguiente panorama:

\section{Sobre la salud y la educación sexual}

E: ¿Reciben información sobre sexualidad y métodos anticonceptivos?

Aos: ¡Sí!

Aa: Lo vimos hace dos meses, más o menos.

Aa: Desde primero, el maestro de Biología nos empezó a platicar sobre eso.

Ao: Sí, llevan un buen seguimiento de esto.

$\mathrm{E}:$ ¿Consideran suficiente esta información?

Aos: ¡Sí! 
Aa: Además, pidió que firmaran los papás para que estuvieran enterados de que nos iban a hablar de eso. Si ellos autorizaban que viéramos ese tema, todos juntos, y ya la maestra recogió los recados y empezaron a darse. Fue como un mes entero.

Ao: Esa clase la impartió una maestra y nosotros tenemos un maestro diferente. No nos tocó su clase.

Aa: Es que tienen a ese maestro, nosotros tenemos aquí a la maestra (...), y en lo personal nos dio la información más completa y clara.

(Grupo D.023)

\section{Sobre el derecho de acceso y uso de las tecnologías de la información}

E: ¿Les dan pláticas sobre tecnologías de la información y su uso?

Aa: Hace poco tuvimos una charla en la que nos hablaron de las redes sociales, cómo usarlas, cómo protegernos, de lo que subimos y así. Si subimos una foto con el uniforme es como muy lógico que vamos en esta secundaria, así que tuviéramos cuidado con la foto.

Ao: Viene seguridad externa, viene la policía municipal para las pláticas.

Aa: En sí la institución o los maestros no, sería más bien por fuera, la policía.

(Grupo A.021)

\section{Sobre la salud y las adicciones}

E: ¿Los orientan sobre el consumo de drogas?

Aos: ¡Sí!

Ao: De hecho, un policía.

Aa: La policía.

Ao: Llegaron a nuestro salón y nos hablaron sobre cada droga y sus consecuencias.

Ao: También vienen de una clínica de rehabilitación.

Aa: Pero muy poquito, ¿no?

Ao: Pero igual, de todas formas, estos temas los abordamos todos los años con alguna maestra.

E: ¿La información es buena?

Aos: Sí.

(Grupo D.025)

Con respecto a la orientación vocacional como parte del derecho a tener una educación de calidad, nos encontramos lo siguiente:

E: ¿Les dan información sobre qué estudiar en el futuro, sobre sus intereses personales?

Aa: Trabajamos los proyectos de vida.

E: ¿Qué hacen en ellos?

Ao: Planteamos una idea a corto, mediano y largo plazo.

E: ¿En ese proyecto ven cuáles son las carreras? ¿Les recomiendan alguna?

Aa: No, esa vez solo nos decían qué carreras hay y cuáles te llamarían la atención. El maestro va viendo tus cualidades y aptitudes; va valorando. Ellos nos decían cuáles carreras venían más apegadas por nuestra forma de ser, pero decir «Ah, estas son las mejores carreras o estas están al tanto», pues no, no es así.

(Grupo E.025)

Uno de los objetivos de la LGDNNA es promover la participación de las adolescentes y los adolescentes en los asuntos de su incumbencia, de acuerdo con su edad, desarrollo cognoscitivo y madurez. Pero desde la perspectiva de la comunidad docente, ¿deben las estudiantes y los estudiantes participar en los asuntos y actividades de la escuela? Las respuestas se pueden apreciar en la tabla 2. 
Tabla 2. Capacidad deliberativa de las adolescentes y los adolescentes en temas escolares, según el profesorado

\begin{tabular}{|c|c|c|c|c|c|}
\hline Etiqueta de valor & Valor & Frecuencia & Porcentaje & $\begin{array}{c}\text { Porcentaje } \\
\text { válido }\end{array}$ & $\begin{array}{c}\text { Porcentaje } \\
\text { acumulado }\end{array}$ \\
\hline Mucha & 1.00 & 61 & 72.62 & 72.62 & 72.62 \\
Algo & 2.00 & 19 & 22.62 & 22.62 & 95.24 \\
Poco & 3.00 & 4 & 4.76 & 4.76 & 100 \\
Nada & 0 & 0 & 0 & 0 & \\
\hline
\end{tabular}

Así, un porcentaje importante de profesoras y profesores estima que el alumnado puede deliberar sobre asuntos de la escuela, postura que refuerzan con expresiones como «son la parte más importante de la escuela» (Profa.21) ${ }^{5}$; «siempre ven las cosas desde otra perspectiva» (Prof.72); «creo que si partimos del interés de los alumnos tendríamos siempre mayor participación, porque estarán desarrollando actividades que les gustan» (Profa.18).

Con todo, las adolescentes y los adolescentes argumentan que en algunas actividades no se toman en cuenta sus opiniones, particularmente con respecto al reglamento escolar. El siguiente fragmento ejemplifica una postura constante en los grupos de trabajo:

$\mathrm{E}: ¿$ ¿Ustedes participan en la elaboración de las reglas?

Ao: ¿Que si participamos haciéndolas?

E: Sí.

Ao: Sí, solo que ellos ponen el $90 \%$ y nosotros el $10 \%$. Bueno, un $5 \%$.

$\mathrm{E}$ : ¿Les gustaría participar más en la elaboración del reglamento?

Ao: Sí, porque ellos solo ponen reglas a favor.

E: ¿Qué les parecen esas reglas que pone la escuela?

Aa: Son rígidas y absurdas algunas veces. Que los hombres deben traer el cabello corto, o sea, no estudias con el cabello. No le dices «como traes el cabello así no vas a poder leer» y que les nieguen estudiar por eso. O sea, vienen a aprender y les prohíben eso solamente por su apariencia... Eso sí es absurdo.

Ao: Como tú te pintas, como te vistes, como traes tu cabello, es como tú eres.

Aa: Te quieren formar como algo que tú no eres; tú te vas a formar según te sientas, tu estilo no te lo pueden cambiar.

(Grupo F.041)

Estas tensiones entre adolescentes y escuela se ilustran también con la dependencia hacia el teléfono celular que, según Ruiz-Palmero, Sánchez-Rodríguez y Trujillo-Torres (2016), ha aumentado considerablemente entre la juventud. ¿Cómo se vive esto en las escuelas? En el siguiente diálogo se expresa la tensión que genera el asunto y que confronta el orden que encarnan profesoras y profesores con el derecho a comunicarse.

E: ¿Qué pasa cuando usan el teléfono en el salón?

Ao: Te lo quitan y solo te lo dan cuando venga tu papá o hasta que acabe el curso; eso depende del maestro.

Aa: Te dicen: «No te lo voy a entregar hasta que termine la clase».

Ao: Hasta que vengan tus papás.

Ao: Pero luego se pasan hasta ocho o quince días...

Aa: Eso no debe ser. Y si es una emergencia, tu mamá te habla, puede ser muy urgente y

5 Los códigos hacen referencia al género y al número de profesor. 
necesitas contestar, necesitas usarlo. Hay veces que preguntas: «Maestra, ¿puedo usarlo?», y la respuesta es «iNo!». «Pero es que es una emergencia», replica uno. «¡No puedes! Esta es mi clase». «Es que es en serio; vea, es mi mamá, si quiere se la paso». «¡No, no puedes en mi clase!».

Aa: Una maestra de Matemáticas les quitó el celular a muchas personas de mi salón. Una vez casi pierde un teléfono y a mí me perdió mis audífonos porque los recogía por una semana. Pero ella no sabe dónde los dejaba ni nada, y ya después te decía: «Yo no sé dónde está, pero es tu culpa porque lo utilizaste».

(Grupo D.052)

Si bien las estudiantes y los estudiantes concuerdan con medidas para su uso dentro del aula, consideran que su opinión debe tenerse en cuenta:

E. ¿Ustedes creen que debe haber un reglamento para el uso del celular?

Ao: Sí.

Ao: No solo lo que los maestros dijeran...

Ao: $\mathrm{O}$ entre los dos...

Aa: Entre los dos, porque si nosotros decimos cómo, vamos a abusar, y si los maestros dicen cómo, pues no... Más bien sería que todos estuviéramos de acuerdo.

(Grupo B.049)

Debido a que es un tema muy controvertido, preguntamos al profesorado si justifican el uso de celulares en la escuela. Su postura se muestra a renglón seguido (tabla 3).

Tabla 3. Justificación del uso de celulares en la escuela, según el profesorado

\begin{tabular}{|c|c|c|c|c|c|}
\hline Etiqueta de valor & Valor & Frecuencia & Porcentaje & $\begin{array}{c}\text { Porcentaje } \\
\text { válido }\end{array}$ & $\begin{array}{c}\text { Porcentaje } \\
\text { acumulado }\end{array}$ \\
\hline (S/R) Sin respuesta & 0 & 2 & 2.38 & 2.38 & 2.38 \\
Mucho & 1 & 11 & 13.10 & 13.10 & 15.48 \\
Algo & 2 & 23 & 27.38 & 27.38 & 42.86 \\
Poco & 3 & 20 & 23.81 & 23.81 & 66.67 \\
Nada & 4 & 28 & 33.33 & 33.33 & 100 \\
\hline \multicolumn{2}{r|}{ Total } & $\mathbf{8 4}$ & $\mathbf{1 0 0}$ & $\mathbf{1 0 0}$ & $\mathbf{1 0 0}$ \\
\hline
\end{tabular}

Si sumamos los juicios de valor «poco» y «nada», un número significativo de profesoras y profesores no aprueba el uso del celular en la escuela. Tal negativa obedece a juicios como estos: «le dan mal uso en el transcurso que están en la escuela» (Profa.28); «no justifico su uso, en la escuela debemos formar hábitos» (Prof.54); «se entretienen en las redes sociales» (Profa.34); «por el mal uso que le están dando a la tecnología, ya que la mayoría se distrae con ellos durante las clases» (Prof.08).

¿De qué otra manera se manifiesta en la escuela la controversia entre derechos y obligaciones? Sabemos que en la escuela alumnas y alumnos en ocasiones asumen conductas que, reglamentadas o no, son rechazadas y, en ocasiones, motivo de expulsión. La postura de las docentes y los docentes se muestra en la tabla siguiente (tabla 4). 
Tabla 4. Conductas que motivan la expulsión de estudiantes, según el profesorado

\begin{tabular}{|l|c|c|c|c|c|c|}
\cline { 2 - 8 } \multicolumn{2}{c|}{} & \multicolumn{5}{c|}{ Valor de la etiqueta } \\
\cline { 2 - 8 } \multicolumn{1}{c|}{} & \multicolumn{2}{c|}{ Átem } & N.uerdo & \multicolumn{2}{c|}{ Desacuerdo } & \multicolumn{2}{c|}{ No sé } \\
\hline Ingiera drogas & ${ }^{\circ}$ & $\%$ & N. $^{\circ}$ & $\%$ & N. $^{\circ}$ & $\%$ \\
\hline Ingiera bebidas embriagantes & 46 & 54.76 & 27 & 32.14 & 9 & 10.71 \\
\hline Fume cigarrillos & 34 & 40.48 & 38 & 45.24 & 10 & 11.90 \\
\hline Resulte embarazada & 17 & 20.24 & 52 & 61.90 & 11 & 13.10 \\
\hline Tenga relaciones sexuales con algún o alguna compañera & 20 & 23.81 & 51 & 60.71 & 9 & 10.71 \\
\hline Sea violento con sus compañeros & 50 & 2.38 & 25 & 59.52 & 7 & 29.76 \\
\hline No respete la disciplina impuesta por la escuela & 40 & 47.62 & 33 & 39.29 & 7 & 8.33 \\
\hline Se enfrente a los profesores & 44 & 52.38 & 26 & 30.95 & 9 & 10.71 \\
\hline Asuma conductas homosexuales & 2 & 2.38 & 75 & 89.29 & 4 & 4.76 \\
\hline Agreda verbalmente a sus compañeros o compañeras & 14 & 16.67 & 60 & 71.43 & 7 & 8.33 \\
\hline Reprobación sistemática & 15 & 17.86 & 59 & 70.24 & 6 & 7.14 \\
\hline Vean pornografía en clase & 23 & 27.38 & 43 & 51.19 & 14 & 16.67 \\
\hline
\end{tabular}

Aquí el consumo de drogas y bebidas dentro de la escuela, así como no respetar la disciplina escolar y enfrentarse a profesoras y profesores, constituyen las frecuencias más altas que motivan la expulsión de estudiantes. Ante su presencia, la determinación de la escuela es corregirlas, con el argumento de ser una medida acertada; sin embargo, a veces las autoridades escolares expulsan a las jóvenes y los jóvenes que cometen infracciones como forma de castigo, oponiéndose a su derecho a la educación. Las adolescentes y los adolescentes reconocen abiertamente lo inadecuado de esas conductas al decir que «deben aplicarse medidas correctivas» (Gpo. C. 073) y que «todos tienen derecho a una oportunidad» (Gpo. A.077), pero «no se les debe expulsar» (Gpo. B.067). Exploramos algunos casos hipotéticos:

Si a algunos de sus compañeros los encontraran manteniendo relaciones sexuales:

E: ¿Si encuentran a compañeros teniendo relaciones sexuales?

Aos: (Risas)

Aa: Foto pa'l Face...

Ao: Darles condones.

E: ¿Deben expulsarlos? ¿Lo consideran grave?

Aa: Pues sí, porque es una falta de respeto.

E: ¿Ustedes creen que los deben expulsar, de acuerdo con las normas de la escuela?

Aa: No correr, no más suspender.

Aa: Les deben dar oportunidades.

Ao: Sin decirle a nadie, que quede eso ahí...

Ao: No correrlos. Si yo los viera, me iba de filo, sin tomarlos en cuenta.

Aos: (Risas).

Aa: Ya es algo íntimo, es muy su bronca; si lo quieren hacer ahí, pues...

(Grupo A.078) 
Si alguno de sus compañeros es sorprendido con marihuana:

E: Y si le encuentran un cigarro de marihuana, ¿expulsión?

Ao: Sería apoyo...

Ao: Apoyo.

Aa: Hablarles a los papás y trabajar entre todos, porque puede tener problemas.

E: Entonces, ¿expulsión o apoyo?

Ao: Una rehabilitación, ¿no? Para que deje eso.

Aa: Es que no sirve de nada; ¿cómo expulsar al niño?

Aa: No se va a curar, y va a ir a otra escuela a fumar o hacerse adicto.

Aa: Lo vas a perjudicar más si lo corres.

Ao: De hecho, va a empeorar.

(Grupo D.083)

En la conjetura de que una de sus compañeras saliera embarazada:

E: ¿Qué hacer si una niña sale embarazada?

Aa: Pues es lo mismo. O sea, no vas a expulsar a alguien solo porque está embarazada; van a pensar que no puede seguir estudiando porque afectaría a la escuela. Mejor ver el problema: ¿por qué salió embarazada? Quizás tiene problemas en su casa o alguien la violó. Yo le brindaría apoyo a ella y al bebé, ya que si ella quiere estudiar puede seguir haciéndolo.

E: ¿Están de acuerdo con su compañera?

Aos: ¡Sí!

(Grupo E.093)

Es evidente que la expulsión no es una posibilidad para la comunidad estudiantil porque sus integrantes piensan que es posible resolver cualquier problema con apoyo y orientación.

Finalmente, después de deliberar sobre estos asuntos, y reconociendo que las adolescentes y los adolescentes tienen la suficiente capacidad para ejercer sus derechos y definir sus deseos (LópezContreras, 2015), les pedimos calificar el actuar de la escuela como protectora y promotora de sus derechos. Los resultados, aunque no son calificados como reprobatorios, en una escala que va del 0 al 10, sí indican que falta mucho por hacer en cuanto al ejercicio efectivo de sus derechos (tabla 5).

Tabla 5. Calificaciones obtenidas por la escuela como promotora de DH, según las personas adolescentes

\begin{tabular}{|c|c|c|c|}
\hline Escuela & Calificación & Escuela & Calificación \\
\hline $\mathrm{A}$ & 6 & $\mathrm{D}$ & 8 \\
\hline $\mathrm{B}$ & 8 & $\mathrm{E}$ & 7 \\
\hline $\mathrm{C}$ & 7 & $\mathrm{~F}$ & 7 \\
\hline
\end{tabular}

Así mismo, como una valoración general sobre la escuela, preguntamos al profesorado si recibe capacitación sobre los DH de las adolescentes y los adolescentes, incluyendo la LGDNNA. Los resultados confirman la poca atención que las autoridades educativas otorgan al tema como parte de la capacitación y actualización permanentes del personal docente. Nunca y rara vez son las respuestas de profesoras y profesores que logran un mayor porcentaje de aparición, alcanzando la primera un $50 \%$ y la segunda un $34.52 \%$, mientras que el porcentaje de los docentes que dice que Siempre o frecuentemente reciben cursos de capacitación alcanza la cifra de $13.09 \%$.

\section{Discusión}

De acuerdo con estos resultados, podemos apuntar que los agentes entrevistados tienen un saber marginal sobre la LGDNNA. Esto significa que el entendimiento y el disfrute de los DH no 
se fundamentan en el conocimiento de la ley, sino en una especie de intuición moral que configura, tanto en el fondo como en la forma, una serie de prácticas caracterizadas por la poca atención al ejercicio efectivo de los derechos en la escuela. Trayendo a colación a Rorty (1998), estos resultados confirman que la emergencia de la cultura de los DH en nuestra sociedad parece que no se debiera a un incremento del conocimiento moral, sino a frecuentes sucesos de historias tristes y sentimentales, insuficientes por sí mismos para la formación del ethos, condición necesaria para tomar decisiones morales razonadas.

Aristóteles (2012) asegura que los seres humanos deliberamos sobre los medios, no sobre los fines. Así, el medio formal empleado por la escuela para promover la cultura de los DH en el aula es plasmar contenidos en una materia, pero este es un medio insuficiente, como lo expresan alumnas y alumnos. No cuestionamos que ciertas materias traten sus obligaciones y derechos con las adolescentes y los adolescentes. Mejor dicho, el asunto central es que el ejercicio efectivo de los DH se asocia a prácticas, rutinas y costumbres que no garantizan la observancia de aquellos en la escuela. Como hemos sugerido, para un ejercicio efectivo es menester una comprensión reflexiva y reiterada sobre el significado de los $\mathrm{DH}$, recuperando la sensibilidad moral de profesoras, profesores y estudiantes, combatiendo la indiferencia moral instalada intermitentemente en las escuelas debido, entre otras cosas, a la cosificación de la tarea docente.

Igualmente, advertimos que el profesorado mantiene una postura paradójica. Por un lado, visualizan a las jóvenes y los jóvenes como agentes morales capaces de reflexionar sobre sus derechos y obligaciones; pero, por otro lado, son considerados como pacientes morales, sujetos que solo reciben ayuda, órdenes, reglas y castigos, incapaces de participar deliberativamente en la resolución de sus problemas. Esta segunda posición revelaría por qué la comunidad estudiantil piensa que la escuela tiene obligaciones con ella, pero el estudiantado no con la escuela. Así mismo, explicaría por qué profesoras y profesores aprueban a priori que determinadas conductas merecen la expulsión de las estudiantes y los estudiantes, sin considerar los problemas concretos y particulares que viven ellas y ellos.

Precisamente, según nuestra reflexión, el desconocimiento de la LGDNNA se vincula en forma directa con el ejercicio no eficaz de los $\mathrm{DH}$ : en la narrativa de profesoras, profesores y estudiantes se advierte ese desconocimiento que implica, en mayor o menor medida, la inclinación de la comunidad docente a vulnerar los derechos de las estudiantes y los estudiantes, al tiempo que estos tienden a no exigirlos, lo cual se observa nítidamente en el caso de las disposiciones tomadas sobre el empleo del celular en la escuela. No debemos desconocer que las adolescentes y los adolescentes utilizan cotidianamente las tecnologías de la información y la comunicación (Gil-Antón, 2013), siendo el celular el medio por excelencia para comunicarse, informarse y divertirse. Para ellas y ellos, el celular es una herramienta vital en sus actividades diarias, pero la constante prohibición del profesorado es vista como una intromisión a sus pertenencias personales, lo que provoca una tensión que enrarece el ambiente escolar, sin abonar a la construcción de la sensibilidad moral de las jóvenes y los jóvenes, elemento particularmente importante en la construcción de una cultura de los DH.

Bázquez-Ruiz (2013) menciona que la escuela presenta dificultades para establecer límites claros y precisos respecto a pautas de conducta y comportamientos «a la hora de decidir entre consentir o corregir» (p. 47). La poca claridad de las reglas (o su ausencia) sobre el uso del celular en la escuela muestra que la comunidad escolar desconoce cómo construir mecanismos racionales de entendimiento, idea que nos remite nuevamente a sostener el poco conocimiento del espíritu de la LGDDNA. Ante los nuevos tiempos, las docentes y los docentes deben encontrar medidas adecuadas y prudentes para un empleo racional del teléfono en el aula. ¿Acaso no consiste en eso la sabiduría docente? ¿Acaso no es eso lo que la sociedad le demanda? Es difícil discernir si se deben tomar medidas drásticas o ser condescendientes ante ciertas actitudes de alumnas y alumnos; sin embargo, en términos aristotélicos, ni el exceso ni el defecto son las reglas que hay que seguir. Aquí, si se desean formas más democráticas de relacionarse en la escuela, lo adecuado sería construir conjuntamente una regla, porque cada caso y cada circunstancia particular son diferentes. 
Es preciso indicar que las normas son legítimas cuando las personas a quien se dirigen se sienten creadoras racionales de estas (Habermas, 2001). Este principio no se cumple en relación con los reglamentos escolares, porque encarnan qué está permitido y qué no, quién decide y quién obedece. Escuchando a entrevistadas y entrevistados, podemos afirmar que este es un instrumento de castigo y no de disuasión; transparenta la profunda incomprensión de la escuela sobre muchos de los problemas que les atañen. Esto también explica por qué, para algunas estudiantes y algunos estudiantes, el reglamento simboliza la anulación de sus voces, reduciéndose a ser un dispositivo útil para sancionar; de ahí la razón del continuo cuestionamiento a las normas escolares. Por eso confirmamos la necesidad de cultivar la buena deliberación del alumnado en las disposiciones que pretenden regular sus conductas en la escuela, propiciando acuerdos razonados para la convivencia escolar, educándolos como sujetos de derechos y no sujetos de castigo. Dado que no somos exclusivamente seres racionales, sino también emotivos (Camps, 2011), se requiere un ejercicio profundo de sensibilización para el conocimiento de los DH porque, según Etzioni (1999), forja el carácter prudente y temperante que toda comunidad debe cultivar.

Ese ejercicio de sensibilización se enfrenta en el aula a lo que podemos llamar una episteme en torno a los $\mathrm{DH}$ que arraiga el pensamiento y los modos de actuar de las profesoras y los profesores, tejiendo una compleja red de creencias y convicciones que conduce a resistirse al reconocimiento de los derechos, a permanecer indiferentes o a mostrar preocupación por su observancia, configurando una práctica de baja intensidad que determina su ejercicio al criterio de cada docente. Además, observamos que las valoraciones del profesorado se mueven entre la confusión y la indiferencia, entre el temor y la omisión involuntaria de sus obligaciones morales en el salón de clases, llevando implícito un sentimiento de victimización asociado a un ejercicio profesional que desdeña sus derechos laborales. Frases como esta lo atestiguan: «Los derechos de los alumnos sí y los de nosotros cuándo» (Prof.05). En consecuencia, la lección es la siguiente: si queremos agentes promotores de los DH en la escuela, activemos mecanismos para revertir la práctica docente que prima facie parece envuelta en la indiferencia moral.

\section{Conclusión}

Finalmente, conviene hacer hincapié en que no encontramos un ambiente totalmente desalentador en las escuelas. Sí existen interpelaciones a ciertas actitudes de la comunidad docente y las autoridades escolares, pero las estudiantes y los estudiantes reconocen que se la pasan bien en las aulas. Ellas y ellos concluyen esto después de un proceso deliberativo, mediante el cual aprueban a sus escuelas en materia de promoción y respeto de sus DH. Entonces, ¿es la escuela capaz o no de promover los DH? Se puede decir que, pese a las adversidades, profesoras, profesores y estudiantes están dispuestos a encontrar mecanismos para convertir el aula en un lugar más apacible y disfrutable para toda la comunidad. Por esto, la capacitación docente del profesorado y la promoción de la LGDDNA entre las adolescentes y los adolescentes son indudablemente una alternativa real para los DH en la escuela.

Por último, reiteramos que este acercamiento no arroja resultados concluyentes sobre el ejercicio efectivo de los DH en la escuela, toda vez que su alcance es exploratorio y, por tanto, limitado. Se requieren estudios más amplios y sistemáticos que posibiliten una caracterización integral del ambiente escolar. Así mismo, reconocemos que muchas de las reflexiones adolescentes pertenecen a una dimensión subjetiva de percepciones individuales, de experiencias y vivencias construidas dentro del salón de clases y determinadas por las formas en que el colectivo escolar las interpreta. Por su parte, la comunidad docente reclama que el tratamiento de los DH en la escuela debe reconsiderar sus condiciones y responsabilidades frente a las alumnas y los alumnos, tema poco analizado en nuestro contexto. En consecuencia, una tarea pendiente consiste en determinar dichas condiciones para convertir las aulas en espacios apropiados para el ejercicio efectivo de los $\mathrm{DH}$. Esto precisa combinar acciones y actitudes difíciles de generar por el momento desde nuestra tradición educativa, si se le deja la responsabilidad exclusiva al profesorado. Quizá sea menester empezar por atribuirle y 
encontrarle un sentido diferente a la escuela, lo cual implica que, como sociedad, le debemos otorgar un respeto especial que nada ni nadie puede vulnerar.

\section{Lista de referencias}

Anaya, A. (2008). Hacia una metodología para el diagnóstico y la medición de la situación de los derechos humanos en México. En J. C. Arjona \& G. Guzmán (Comps.), Hacia una metodología para la medición del cumplimiento de los derechos humanos en México (pp. 55-75). Ciudad de México: Universidad Iberoamericana.

Aristóteles (2012). Ética nicomáquea. Ciudad de México: Unam.

Bauman, Z., \& Donskis, L. (2015). Ceguera moral: la pérdida de sensibilidad en la modernidad líquida. Ciudad de México: Paidós.

Bázquez-Ruiz, F. (2013). Dialéctica de los derechos humanos. Madrid: Dykinson.

Bernal, C. (2008). El derecho de los derechos. Bogotá, D. C.: Universidad Externado de Colombia.

Calvinho, G. (2012). El proceso con derechos humanos: método de debate y garantía frente al poder. Bogotá, D. C.: Universidad del Rosario.

Camps, V. (2011). El gobierno de las emociones. Barcelona: Herder.

Comisión Nacional de los Derechos Humanos. (2015). Ley general de los derechos de niñas, niños y adolescentes. Ciudad de México: Autor. Recuperado de: http://200.33.14.34:1033/archivos/pdfs/ lib_LeyGralCuidadoInfantil.pdf.

Comisión Nacional de los Derechos Humanos. (2018). Informe de actividades. Del $1^{\circ}$ de enero al 31 de diciembre de 2017. Ciudad de México: Autor. Recuperado de: http://informe.cndh.org.mx/ images/uploads/menus/30110/content/files/Informe_cndh_2017.pdf.

Comisión Nacional de los Derechos Humanos, \& Centro de Investigaciones y Estudios Superiores en Antropología Social. (2017). Informe especial adolescentes: vulnerabilidad y violencia. Ciudad de México: Autores. Recuperado de: http://www.cndh.org.mx/sites/all/doc/Informes/Especiales/ Informe_adolescentes_20170118.pdf.

Córdova, L., Flores, J. I., Alejandre, O., \& Vázquez del Mercado, S. (2015). Encuesta Nacional de Niños, Adolescentes y Jóvenes. Ciudad de México: Unam. Recuperado de: http://www. losmexicanos.unam.mx/culturapolitica/encuesta_nacional.html.

Durán-Strauch, E. (2017). Derechos de niños y niñas: del discurso a la política local. Revista Latinoamericana de Ciencias Sociales, Niñez y Juventud, 15(2), pp. 879-891. doi:10.11600/169 $2715 x .1520623062016$.

Etzioni, A. (1999). La nueva regla de oro: comunidad y moralidad en una sociedad democrática. Barcelona: Paidós.

Flick, U. (2007). Introducción a la investigación cualitativa. Madrid: Morata.

Fuentes, M. L., González, M., Padrón, M., \& Tapia, E. (2015). Conocimientos, ideas y representaciones acerca de niños, adolescentes y jóvenes. ¿Cambio o continuidad? Encuesta Nacional de Niños, Adolescentes y Jóvenes. Ciudad de México: Unam. Recuperado de: http://www.losmexicanos. unam.mx/ninosadolescentesyjovenes/index.html.

García-Cívico, J. (2010). ¿Qué es un indicador de derechos humanos y cómo se utiliza? Revista Derechos y Libertades, 24(II), 179-219.

Garzón, E. (2005). Lo íntimo, lo privado y lo público. Cuadernos de Transparencia, 6, 5-37.

Gil-Antón, A. M. (2013). El derecho a la propia imagen del menor en internet. Madrid: Dykinson. Recuperado de: https://ebookcentral-proquest-com.wdg.biblio.udg.mx/lib/guadalajarasp/reader. action?docID $=3218945$ \& query $=$ El $\% 20$ derecho $\% 20 \mathrm{a} \% 201 \mathrm{a} \% 20$ propia $\% 20$ imagen $\% 20$ del $\% 20$ menor\%20en\%20internet.

González, M. (2016). La Constitución mexicana vista a la luz de los modelos de tratamiento jurídico de la infancia y adolescencia. En L. R. Guerrero \& C. M. Pelayo (Coords.) Cien años de la 
Constitución mexicana: de las garantías individuales a los derechos humanos (pp. 339-356). Ciudad de México: Unam.

Habermas, J. (2001). Facticidad y validez, sobre el derecho y el Estado democrático de derecho en términos de teoría del discurso. Madrid: Trotta.

Hernández, J., Cardona-Arango, D., \& Segura-Cardona, A. M. (2018). Construcción y análisis de un índice de vulnerabilidad social en la población joven. Revista Latinoamericana de Ciencias Sociales, Niñez y Juventud, 16(1), 403-412. doi:10.11600/1692715x.16125.

Hernández, R., Fernández, C., \& Baptista, P. (2014). Metodología de la investigación. Ciudad de México: McGraw-Hill.

Instituto Nacional para la Evaluación de la Educación. (2016). La educación obligatoria en México. Informe 2016. Ciudad de México: Autor. Recuperado de: http://www.inee.edu.mx/images/ stories/2016/informe 2016/Informe2016-1pdf.pdf.

López-Contreras, R. E. (2015). Interés superior de los niños y niñas: definición y contenido. Revista Latinoamericana de Ciencias Sociales, Niñez y Juventud, 13(1), 51-70. doi:10.11600/169271 5x.1311210213

Lozano-Vicente, A. (2016). Los derechos del niño: cuestiones sobre su fundamentación. Revista Latinoamericana de Ciencias Sociales, Niñez y Juventud, 14(1), 67-79. doi:10.11600/169271 $5 x .1413290515$.

Matus, E. (2013). México ante la restitución internacional de menores. Ciudad de México: Unam.

Nussbaum, M. (2006). Las fronteras de la justicia. Barcelona: Paidós.

Ragin, Ch. (2007). La construcción de la investigación social. Bogotá, D. C.: Siglo del Hombre.

Ravetllat-Ballesté, I. (2016). Precongresos mundiales por los derechos de la infancia y la adolescencia, 2012-2014. Revista Latinoamericana de Ciencias Sociales, Niñez y Juventud, 14(2), 899-911. do i:10.11600/1692715x.14201110815.

Rorty, R. (1998). Derechos humanos, racionalidad y sentimentalidad. En S. Shute \& S. Hurley, De los derechos humanos (pp. 117-136). Madrid: Trotta.

Ruiz-Palmero, J., Sánchez-Rodríguez, J., \& Trujillo-Torres, J. M. (2016). Utilización de internet y dependencia a teléfonos móviles en adolescentes. Revista Latinoamericana de Ciencias Sociales, Niñez y Juventud, 14(2), 1357-1369. doi:10.11600/1692715x.14232080715.

Sáez, J. M. (2017). Investigación educativa: fundamentos teóricos, procesos y elementos prácticos. Madrid: Uned. Recuperado de: https://ebookcentral-proquest-com.wdg.biblio.udg.mx/lib/guadalajarasp/reader.action?docID $=4870247 \&$ query $=$ Investigaci $\% \mathrm{C} 3 \% \mathrm{~B} 3 \mathrm{n} \% 20$ Educativa:\%20fundamentos $\% 20$ te $\% \mathrm{C} 3 \%$ B3ricos, $\% 20$ procesos $\% 20 \mathrm{y} \% 20$ elementos $\% 20 \mathrm{pr} \% \mathrm{C} 3 \% \mathrm{~A} 1 \mathrm{cticos}$.

Secretaría de Educación Pública. (2017). Modelo educativo para la educación obligatoria. Ciudad de México: Autor. Recuperado de:

https://www.gob.mx/cms/uploads/attachment/file/198738/Modelo_Educativo_para_la_ Educacio_n_Obligatoria.pdf.

Silva, D. (2017). Experiencia narrativa. Adolescentes institucionalizados por protección. Barcelona: UOC. Recuperado de: https:/ebookcentral-proquest-com.wdg.biblio.udg.mx/lib/guadalajarasp/ reader.action?docID $=4824486 \&$ query $=$ Experiencia $\% 20$ narrativa. $\% 20$ Adolescentes $\% 20$ institucionalizados $\% 20$ por $\% 20$ protecci $\% \mathrm{C} 3 \% \mathrm{~B} 3 \mathrm{n}$.

Silva, C., \& Martínez-Guzmán, M. L. (2017). El self adolescente desde la perspectiva contextual: pobreza, viviendas sociales, apoyo parental y participación. Revista Latinoamericana de Ciencias Sociales, Niñez y Juventud, 15(1), 117-130. doi:10.11600/1692715x.1510605022016.

Suárez, M. (2005). El grupo de discusión. Una herramienta para la investigación cualitativa. Barcelona: Laertes.

Yuni, J. A., \& Urbano, C. A. (2014). Técnicas para investigar: recursos metodológicos para la preparación de proyectos de investigación. Córdoba: Brujas. 